\title{
Preparation of Antioxidant and Evaluation of the Antioxidant Activities of Antioxidants Extracted From Sugarcane Products
}

\author{
Fansheng Kong ${ }^{1,2,3}$, Shujuan $\mathrm{Yu}^{1,3, *}$, Feng Zeng ${ }^{1}$, Xinlan $\mathrm{Wu}^{1}$ \\ ${ }^{1}$ College of Light Industry and Food Sciences, South China University of Technology, Guangzhou, China \\ ${ }^{2}$ College of Pharmacy, Guangdong Pharmaceutical University, Guangzhou, China \\ ${ }^{3}$ Guangdong Province Key Laboratory for Green Processing of Natural Products and Product Safety, Guangzhou, China \\ *Corresponding author: shujuanyu8@gmail.com
}

Received August 08, 2015; Revised August 19, 2015; Accepted September 09, 2015

\begin{abstract}
Background: Sugarcane is widely consumed by people of the tropics and subtropics. Antioxidants in concentrated sugarcane extracts extracted from sugarcane products using resins are highly stable, and their antioxidant activity is not significantly reduced by prolonged heating or clarification. Methods and Results: This study investigated the extraction processing and evaluated the antioxidant action (DPPH, ABTS, and FRAP) of sugarcane extracts. The total phenolic content and the phenolic composition of sugarcane extracts were analyzed. Our results showed that the sugarcane extracts were potential source of antioxidant compounds. The total phenolic content in sugarcane extracts was $0.179 \pm 0.003 \mathrm{mg}$ equivalent/gram extracts. The major phenolic acids in sugarcane extracts were identified and quantified using high performance liquid chromatography (HPLC). The content of gallic acid, chlorogenic acid, caffeic acid, and ferulaic acid in sugarcane extracts were $(0.87 \mathrm{mg} / \mathrm{g}),(1.77 \mathrm{mg} / \mathrm{g})$, $(11.64 \mathrm{mg} / \mathrm{g})$, and $(10.49 \mathrm{mg} / \mathrm{g})$, respectively. Conclusion: This study provides a basis for further exploitation of the health beneficial resources of sugarcane.
\end{abstract}

Keywords: antioxidant, extract, polyphenol, sugarcane

Cite This Article: Fansheng Kong, Shujuan Yu, Feng Zeng, and Xinlan Wu, "Preparation of Antioxidant and Evaluation of the Antioxidant Activities of Antioxidants Extracted From Sugarcane Products.” Journal of Food and Nutrition Research, vol. 3, no. 7 (2015): 458-463. doi: 10.12691/jfnr-3-7-7.

\section{Introduction}

In the last several decades, there has been an increased interest in identifying free radical scavengers or antioxidants that exhibit positive effects on cardiovascular diseases, cancers, and brain degenerative process due to their significant antioxidant activities [1-6]. These antioxidants supplied by diets, including vitamin C, vitamin E, carotenoids, and several polyphenolic compounds such as flavonoids can prevent these diseases by scavenging free radicals or interfering with DNA binding $[7,8]$. Therefore, antioxidants abundant in foods and plants have been extensively searched and studied because of the important effects on cardiovascular diseases and cancers $[9,10,11]$.

Sugarcane, also known as noble cane, is one of the important industrial crops in the world due to its high sucrose content and low fiber content. Sugarcane is the principal raw material in the sugar industry and approximately $70 \%$ of the world's sugar is made of sugarcane. In addition to sugar production, raw sugarcane and sugarcane juice are commonly consumed by a large number of population in the tropics and subtropics. Furthermore, chewing raw sugarcane is recommended by some medical systems for keeping sound and healthy status [12].

Pigments in sugarcane juice are mainly phenolic compounds. Paton (1992) ${ }^{[13]}$ characterized the phenolic composition of sugarcane and its products and found that the main compounds in sugarcane and its products were phenylpropanoids and flavonoids [14,15]. Nakasone et al. (1996) ${ }^{[16]}$ isolated five antioxidant compounds from kokuto extracts. Takara et al. (2002) ${ }^{[17]}$ identified thirteen antioxidant compounds, including several glycosylated phenolic compounds in kokuto. Some identified compounds exhibited higher antioxidant activity than $\alpha$ tocopherol. Payet, Cheong, and Smadja ${ }^{[18]}$ reported the antioxidant activity of several samples of brown sugar made of sugarcanes and suggested that a number of phenolic acids and flavonoids accounted for at least partially the observed antioxidant activity. Phenolic substances in sugarcane juice may have biological activities ${ }^{[19]}$. For example, an acylated tricin glycoside isolated from sugarcane juice exhibited anti-proliferative activity [20].

While several studies $[19,21,22,23]$ have characterized the antioxidant activity of derivative products presented in the by-products such as molasses, filter mud, and bagasse in sugarcane manufacturing industry, there is a lack of systematic study of the phytochemicals and antioxidant 
activities combined with sugar manufacturing. Characterization of phytochemicals in different parts of sugarcane is useful to understand the potential beneficial effects of sugarcane on human health [24].

In the present study, we evaluated the phenolic contents and antioxidant activities of sugarcane extraction and sugarcane products obtained during sugar extraction. Pigment is one of the important indicators affecting the quality of sucrose in sugar production and de-colorization has been a major problem in sugar industry. Herein, we developed a sugarcane extraction method, which ensured the antioxidant activity of sugarcane extraction with high quality of sugar production.

\section{Materials and Methods}

\subsection{Materials}

Diammonium salt, 1,1'-diphenyl-2-picrylhydrazyl (DPPH), ascorbic acid, 2,2'-azobis-3-ethylbenthiaazoline-6-sulfonic acid (ABTS), Folin Ciocalteu's reagent, gallic acid, chlorogenic acid, caffeic acid , and ferulaic acid were purchased from Aladdin (Shanghai, China). Hydrogen peroxide, potassium phosphate(monobasic and dibasic), sodium carbonate, trolox(6-hydroxy-2,5,7,8-tetramethylchroman-2-carboxyli cacid), 1,1,3,3-tetraethoxypropane, and 2,4,6-tripyridyl-striazine (TPTZ) were purchased from Sigma Chemical Co. (St. Louis, MO,USA). Other chemicals used in the present studies were of the highest quality commercially available in local suppliers.

\subsection{Preparation of Sugarcane Extraction}

Sugarcane (Saccharum spp.) plants of Yuetang 54-474 (green-rind) were collected from Guangdong, People's Republic of China in the sugarcane harvesting season in winter 2012. The pigments in sugarcane indicate strong antioxidant properties. Antioxidants in concentrated sugarcane extracts obtained from sugarcane products (sugarcane molasses), using the macroporous resins and ion exchange resins for extraction [35], have good performance in stability, as even prolonged heating or clarification would not make any reduction of its antioxidant capacity. The antioxidant capacity of sugarcane extracts correlates with their color because the polyphenol components with high antioxidant capacity are responsible for the color of sugarcane bodies. Sugarcane, which is cultivated of large scale in the world for producing sugar, is easier to be obtained for sugarcane extracts compared with other extraction materials. The extraction method was shown in Figure 1.

\subsection{Determination of Total Phenolic Content}

The total phenolic content in the extraction from sugarcane, ethyl acetate, and methanol extracts were assessed using the Folin-Ciocalteau method [25] with slight modification. Briefly, extracts $(10 \mathrm{mg})$, distilled water $(10 \mathrm{~mL})$ and Folin-Ciocalteau's reagent $(0.5 \mathrm{~mL})$ were mixed in a tube, and then $1.5 \mathrm{~mL}$ of $100 \mathrm{~g} / \mathrm{L} \mathrm{Na}_{2} \mathrm{CO}_{3}$ was added to the solution. The reaction mixture was incubated at $25^{\circ} \mathrm{C}$ for $2 \mathrm{~h}$ and the absorbance of the mixture was determined at $763 \mathrm{~nm}$. The sample was tested for three times and a calibration curve with six data points for gallic acid was established. The results were compared to a gallic acid calibration curve and the total phenolic content in the extraction of sugarcane was expressed as mg of gallic acid equivalents per gram of extracts.

\subsection{Identification and Quantification of Phenolic Compounds Using High-performance Liquid Chromatography (HPLC)}

The extraction was identified and quantified using HPLC with UV-vis spectr. The re-dissolved extraction (20 $\mu \mathrm{L} ; 1.0 \mathrm{mg} / \mathrm{mL}$ in methyl alcohol) was analyzed on an analytical HPLC equipment (Agilent 1260), with a Gemini $\mathrm{C}_{18}$ column $(150 \times 4.6 \mathrm{~mm}, 5 \mu \mathrm{m}$ particle size; Phenomenex, Macclesfield, UK). The column was developed by a linear gradient of Solvent A (Acetonitrile: Acetic Acid; 2:98, v/v) and Solvent B (Water: Acetonitrile: Acetic Acid; 78:20:2, v/v) at a flow rate of $0.8 \mathrm{~mL} / \mathrm{min}$ as follows: 0-27 $\min (97 \%-10 \%$ A) / 27-30min ( $10 \%$ A ) / 40 min (10\%-95\% A). Data acquisition and manipulation were performed using Agilent Chemstation B.04.03 DSP1.

\subsection{DPPH free Radical Scavenging Effects}

The DPPH free radical scavenging effects were evaluated using a modified method described by Yamaguchi et al. [26]. Briefly, $0.1 \mathrm{~mL}$ of $10 \mathrm{mM} \mathrm{DPPH}$ solution (in methanol) was added to a test tube containing 0-10 $\mathrm{mg} / \mathrm{mL}$ of extracts. The mixture was shaken vigorously for $15 \mathrm{~s}$ and incubated in the dark at room temperature for $30 \mathrm{~m}$. Methanol was used as a blank control. Vitamin C was used as a positive control. The absorbance at $517 \mathrm{~nm}$ of each sample was measured using a spectrophotometer (752N, Yidian, Shanghai, China). The experiment was performed in triplicate. The percentage of free radical scavenging effects was calculated as Eq. 1:

$$
\begin{aligned}
& \text { DPPH scavenging effects }(\%) \\
& =\left[1-\left(A_{517 n m, \text { sample }} / A_{517 n m, \text { blank }}\right)\right] \times 100
\end{aligned}
$$

where $A_{517 n m \text {, sample }}$ and $A_{517 n m \text {,blank }}$ were the absorbance values at $517 \mathrm{~nm}$ for sample and blank, respectively.

\section{6. $\mathrm{ABTS}^{+}$Free Radical Scavenging Effects}

For ABTS assay, we followed the method described by Miller et al. [27] with slight modification. ABTS ${ }^{++}$radical cation was prepared by passing a $5 \mathrm{mM}$ aqueous stock solution of ABTS (2,2'-azinobis(3-ethylbenzothiazoline-6sulphonicacid adiammonium salt) (Aldrich Chemical Co. Milwaukee, WI, USA) through manganese dioxide (Sigma-Aldrich, St. Louis, MO, USA) on a filter paper. Excess manganese dioxide was removed from the filtrate by passing it through a $0.22 \mu \mathrm{M}$ PVDF syringe filter. The collected solution was then diluted in $5 \mu \mathrm{M}$ phosphate buffered saline (PBS) ( $\mathrm{pH} 7.4$ ) to an absorbance of 0.70 $( \pm 0.02)$ at $734 \mathrm{~nm}$ and pre-incubated at $30^{\circ} \mathrm{C}$ prior to use. Fresh $\mathrm{ABTS}^{++}$radical cation solution was prepared daily. Vitamin C was used as a positive control. The experiment was performed in triplicate. The percentage of ABTS ${ }^{++}$ radical scavenging effects was calculated as Eq. 2: 
ABTS $^{++}$scavenging effect (\%)

$=\left[1-\left(A_{734 n m, \text { sample }} / A_{734 n m, \text { blank }}\right)\right] \times 100$

Eq.2

In which $A_{734 n m \text {, sample }}$ and $A_{734 n m, b l a n k}$ were the absorbance values at $734 \mathrm{~nm}$ for sample and blank, respectively.

\subsection{Ferric-reducing Antioxidant Power (FRAP) Assay}

FRAP assay was conducted according to the method described by Benzie and Strain [28](1996) with slight modification. Briefly, the FRAP reagent was freshly prepared by mixing $100 \mathrm{mM}$ acetate buffer ( $\mathrm{pH}$ 3.6), $10 \mathrm{mM}$ 4,6-tripryridyls-triazine (TPTZ) in $40 \mathrm{mM} \mathrm{HCl}$, and $20 \mathrm{mM}$ ferric chloride in a ratio 10:1:1 (by volume) before FRAP assay. Then, $100 \mu \mathrm{L}$ of extracts and $4.5 \mathrm{~mL}$ of FRAP reagent were transferred into vials and incubated at $37^{\circ} \mathrm{C}$ for $10 \mathrm{~min}$. Absorbance at $593 \mathrm{~nm}$ was measured relative to a reagent blank incubated at $37^{\circ} \mathrm{C}$. The FRAP data for each sample were determined against a standard of known FRAP value, $\mathrm{FeSO}_{4}$ (Sigma-Aldrich, St. Louis, $\mathrm{MO}, \mathrm{USA})$. Results were expressed as $\mathrm{mg} \mathrm{FeSO}_{4} / 100 \mathrm{~g}$.

\section{Results and Discussion}

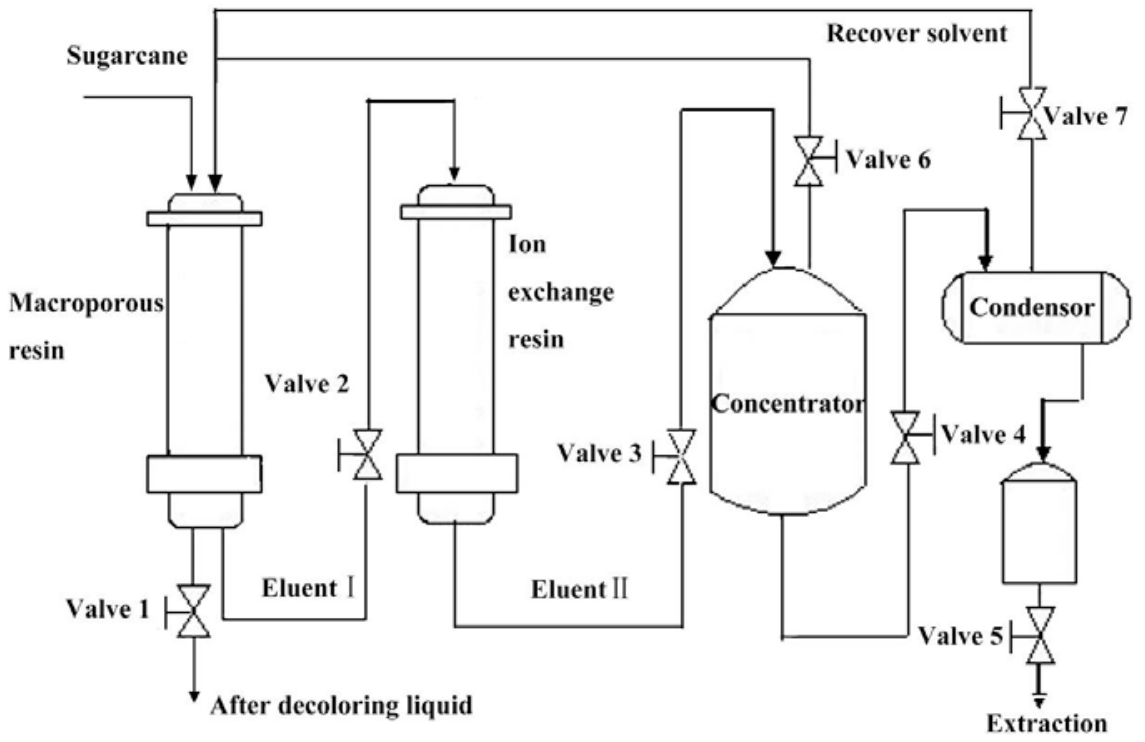

Figure 1. The sugarcane processing and purification systems

\subsection{Total Phenolic Contents}

The total phenolic contents in sugarcane extracts were directly associated with their antioxidant effects. The phenols are potent scavengers of free radicals. The total phenolic contents in sugarcane extracts were $0.179 \pm$ $0.003 \mathrm{mg}$ equivalent/gram extracts.

\subsection{Analysis of HPLC Data}

Identification and quantification of phenolic substances in the eluates were carried out in duplicate. According to retention time (Figure 2), caffeic acid (peak 3: retention time $28.861 \mathrm{~min}$ ) and ferulaic acid (peak 4: retention time $40.264 \mathrm{~min}$ ) were two main phenolic. A number of minor phenolic such as gallic acid (peak 1: retention time 10.559

\subsection{Preparation of Antioxidants from Sugarcane}

Numerous studies have been conducted to extract natural antioxidants, however, the raw plant material used for natural antioxidants are expensive and rare. In addition, the plant material was abandoned in the extraction of antioxidant substances, resulting in the waste of the other components. Sugarcane is a rich source for the extraction of natural antioxidants in the tropics and subtropics with relatively low price. The pigment as antioxidant active substance was extracted with no effect on extraction of sucrose, which made full use of the various components of sugarcane. For sugar production, color is one of the indicators affecting the quality of sucrose and decolorization are the major problems in sugar industry. In the present study, we developed an appropriate method for antioxidant extraction from sugarcane (Figure 1), using macroporous resins and ion exchange resins. This extraction method exhibited fair performance, and the antioxidant capacity of extracts was not significantly reduced by prolonged heating or clarification. This study combined the antioxidant activity of material extraction with sugar manufacturing obtaining higher quality sugar, which has a positive significance. min) and chlorogenic acid (peak 2: retention time 24.478 min) were also detected. These results indicate that sugarcane eluates contained gallic acid $(0.87 \mathrm{mg} / \mathrm{g})$ and chlorogenic acid $(1.77 \mathrm{mg} / \mathrm{g})$, while the content of caffeic acid $(11.64 \mathrm{mg} / \mathrm{g})$ and ferulaic acid $(10.49 \mathrm{mg} / \mathrm{g})$ was almost 10 -fold higher than that of gallic acid $(0.87 \mathrm{mg} / \mathrm{g})$ and chlorogenic acid ( $1.77 \mathrm{mg} / \mathrm{g})$.

We quantified four main phenolic, namely, caffeic acid, ferulaic acid, chlorogenic acid, and gallic acid. Due to their low content, other minor phenolic or unknown compounds were not quantified in the present research. It should be noted that several unknown compounds were not identified in the present study. Therefore, more work need to be done to identify and characterize these unknown compounds in the future. 


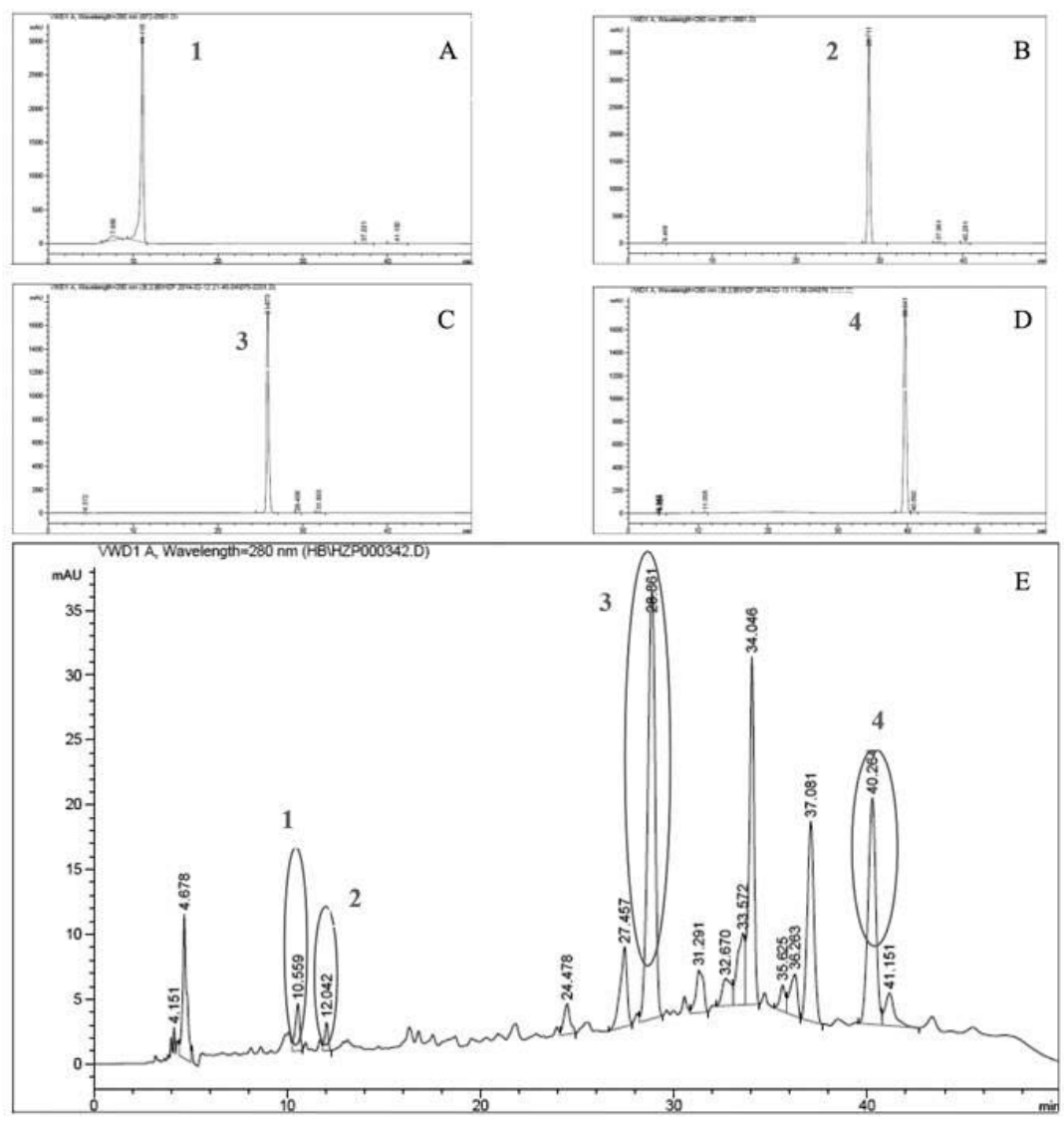

Figure 2. HPLC chromatogram of phytosterol standards (a-d) and sugarcane extracts samples (e). Detection at $280 \mathrm{~nm}$. 1- gallic acid; 2- chlorogenic acid; 3- caffeic acid; 4- ferulaic acid

\subsection{DPPH Radical Scavenging Activity}

DPPH radical is widely used in the estimation of antioxidant activity by its ability of abstracting hydrogen atoms from polyphenols [29]. The extracts exhibited a significant inhibition of DPPH activity in a dosedependent manner. Briefly, a $50 \%$ of inhibition $\left(\mathrm{IC}_{50}\right)$ was achieved when the concentration of sugarcane extracts was $62.84 \pm 0.268 \mu \mathrm{g} / \mathrm{mL}$ (Figure 3 ). The $\mathrm{IC}_{50}$ value of vitamin $\mathrm{C}$ was $6.6 \pm 0.324 \mu \mathrm{g} / \mathrm{mL}$.

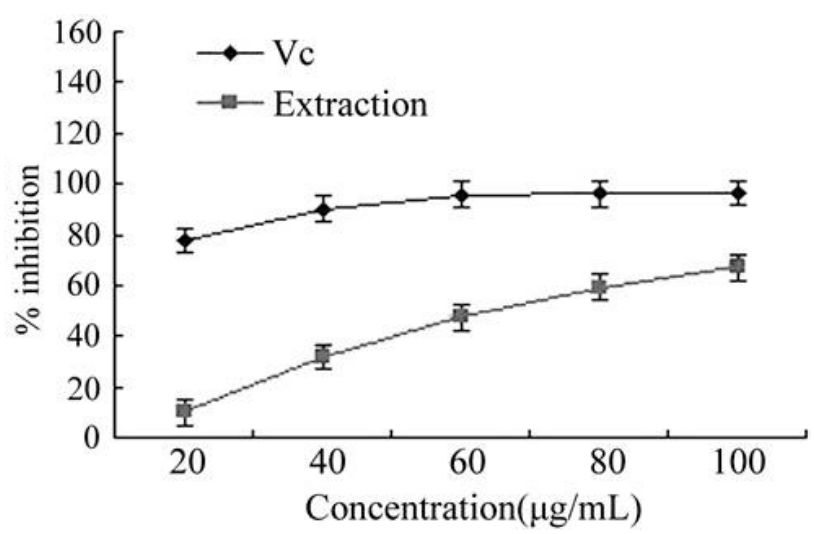

Figure 3. The DPPH scavenging effects of sugarcane extracts of different concentrations $(20-100 \mu \mathrm{g} / \mathrm{mL})$ and vitamin $\mathrm{C}$. The values obtained from triplicate experiments were presented as mean \pm SEM

\subsection{ABTS $^{++}$Radical Scavenging Activity}

In ferrylmyoglobin/ABTS assay, the formation of $\mathrm{ABTS}^{++}$radical due to the reaction between ferrylmyoglobin and ABTS, was delayed with the addition of antioxidant and the inhibition of radicalis formation was measured as lag time in seconds [30]. Free radicals were formed in vivo or taken into body exogenously. The line of defense is the antioxidants that scavenge free radicals to suppress chain initiation and/or break the chain propagation reactions. The extracts exhibited significant inhibition of $\mathrm{ABTS}^{++}$activity in a dose-dependent manner. A $50 \%$ of inhibition $\left(\mathrm{IC}_{50}\right)$ was observed when the concentration of sugarcane extracts was $238.802 \pm 0.139$ $\mu \mathrm{g} / \mathrm{mL}$ (Figure 4). The $\mathrm{IC}_{50}$ value of vitamin $\mathrm{C}$ was $115.910 \pm 0.436 \mu \mathrm{g} / \mathrm{mL}$.

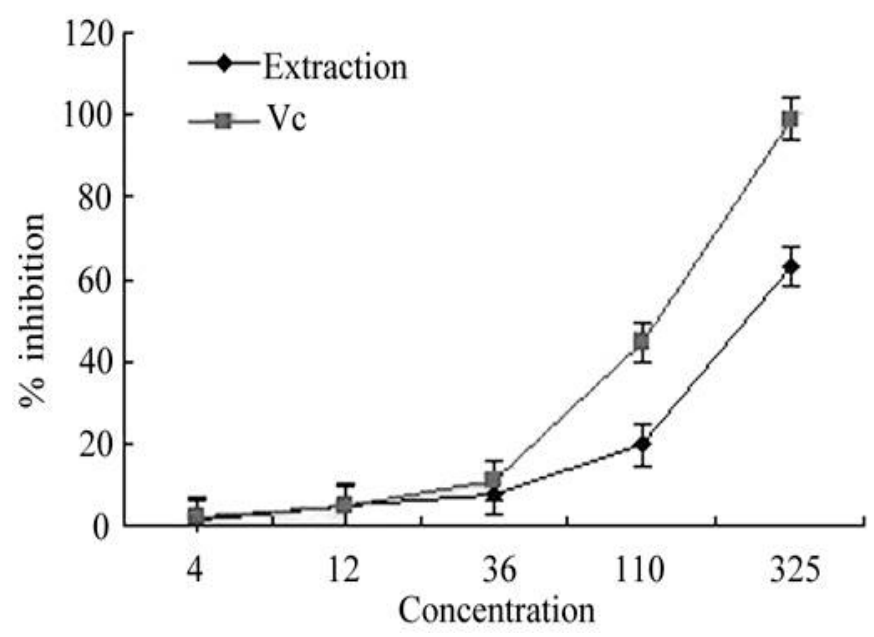

Figure 4. The ABTS scavenging effects of sugarcane extracts of different concentrations $(4-330 \mu \mathrm{g} / \mathrm{mL})$ and vitamin $\mathrm{C}$. The values obtained from triplicate experiments were presented as mean \pm SEM 


\subsection{FRAP}

The FRAP assays was used to evaluate the antioxidant activity of sugarcane extracts. Antioxidants are substances that prevent and/or delay the oxidation of substrates when present at low concentrations. Non-enzymatic antioxidants react with pro-oxidant sand to inactivate them. In this redox reaction, antioxidants act as reductants. In this context, antioxidant power can be referred to as 'reducing ability'. In the FRAP assay, an easily reducible oxidant, Fe(III) was excessively used. Thus, with reduction of the Fe(III)-TPTZ complex by antioxidant, Fe(II)-TPTZ in blue was formed, which can be detected and measured using spectrophotometer at $595 \mathrm{~nm}$ [31]. The first line of defense is preventive antioxidants, which suppress the formation of free radicals. As shown in Fig 5, the extracts exhibited a significant FRAP in a dose-dependent manner, parallel lines were obtained, and the extracts and vitamin $\mathrm{C}$ were tested and different FRAP values with distinct doses were obtained (Figure 5).

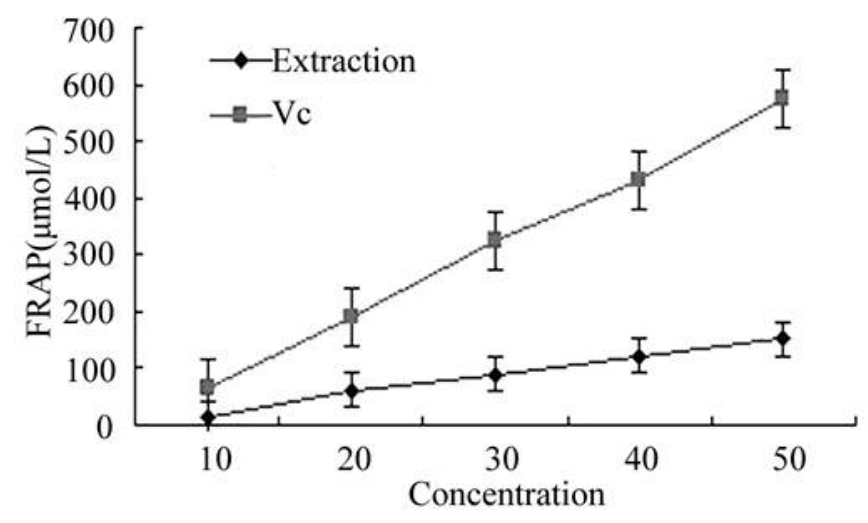

Figure 5. The FRAP scavenging effects of sugarcane extracts of different concentrations $(10-50 \mu \mathrm{g} / \mathrm{mL})$ and vitamin $\mathrm{C}$. The results showed a dose-dependent manner of FRAP

Antioxidant activities were measured in resin eluents obtained using DPPH, ABTS, and FRAP. The DPPH and FRAP assays could be used to evaluate antioxidant activity in sugarcane as both showed high reproducibility [32]. Working solution of DPPH and FRAP was used immediately after preparation, while that of ABTS was kept in dark for $12 \mathrm{~h}$ to generate free radicals from the ABTS salt and then was used within $4 \mathrm{~h}$ [33,34]. Given that the ABTS working solution was not always the same age, the activity of the solution to react with sugarcane extracts might have been different among the determination times.

Sugarcane is appreciated as food in social-economically disfavoured areas of China and other countries. We can find the appropriate extraction method, the antioxidant activity of material extraction combined with sugar manufacturing, while an extracts antioxidant activity to obtain higher quality sugar. This study suggested that the extracts of sugarcane possessed antioxidant activity which might be helpful in preventing or slowing the progress of various oxidative stress-related diseases while obtain higher quality sugar. Further investigation on the isolated component on antioxidant activity may lead to chemical entities for clinical use. All would have a positive significance.

\section{Acknowledgement}

This study was supported by the ministry of science and technology in agriculture science and technology Achievements Transformation Fund Project (No.2013GB23600669), Science and Technology Planning Project of Guang Zhou, China(No.2013B051000015) and the Open Project Program of Provincial Key Laboratory of Green Processing Technology and Product Safety of Natural Products (No.201303).

\section{References}

[1] Conte A., Pellegrini S., Tagliazucchi D.: Synergistic protection of PC12 cells from beta-amyloid toxicity by resveratrol and catechin. Brain Res Bull 2003;62:29-38.

[2] D A Valos A., Bartolom E B., G O Mez-Cordov E S C.: Antioxidant properties of commercial grape juices and vinegars. Food Chem 2005;93:325-330.

[3] Gey K. F.: The antioxidant hypothesis of cardiovascular disease: Epidemiology and mechanisms. Biochem Soc Trans 1990;18:1041-1045.

[4] Lee J., Rennaker C.: Antioxidant capacity and stilbene contents of wines produced in the Snake River Valley of Idaho. Food Chem 2007;105:195-203.

[5] Verzelloni E., Tagliazucchi D., Conte A.: Relationship between the antioxidant properties and the phenolic and flavonoid content in traditional balsamic vinegar. Food Chem 2007;105:564-571.

[6] Visioli F., Borsani L., Galli C.: Diet and prevention of coronary heart disease: The potential role of phytochemicals. Cardiovasc Res 2000;47:419-425.

[7] Craig W. J.: Phytochemicals: Guardians of our health. J Am Diet Assoc 1997;97:S199-S204.

[8] Stoner G. D., Mukhtar H.: Polyphenols as cancer chemopreventive agents. J Cell Biochem 1995;59:169-180.

[9] Abbas S. R., Sabir S. M., Ahmad S. D., Boligon A. A., Athayde M. L.: Phenolic profile, antioxidant potential and DNA damage protecting activity of sugarcane (Saccharum officinarum). Food Chem 2014;147:10-16.

[10] Hertog M. G., Feskens E. J., Hollman P. C., Katan M. B., Kromhout D.: Dietary antioxidant flavonoids and risk of coronary heart disease: The Zutphen Elderly Study. Lancet 1993;342:10071011.

[11] Hertog M. G., Kromhout D., Aravanis C., Blackburn H., Buzina R., Fidanza F., Giampaoli S., Jansen A., Menotti A., Nedeljkovic S., Others: Flavonoid intake and long-term risk of coronary heart disease and cancer in the seven countries study. Arch Intern Med 1995; 155:381-386.

[12] Kadam U. S., Ghosh S. B., De S., Suprasanna P., Devasagayam T., Bapat V. A.: Antioxidant activity in sugarcane juice and its protective role against radiation induced DNA damage. Food Chem 2008;106:1154-1160.

[13] Patton N. H., Duong M.: Sugar cane phenolics and first expressed juice colour. Part II. Concentration of phenolics in sugar cane and colour of first expressed juice. Int. Sugar J 1992;94:205.

[14] Harborne J. B., Hall E.: Plant polyphenols - XII.: The occurrence of tricin and of glycoflavones in grasses. Phytochemistry 1964;3:421-428.

[15] Smith P., Paton N. H.: Sugarcane flavonoids. Sugar Technol. Rev 1985;12:117-142.

[16] Nakasone Y., Takara K., Wada K., Tanaka J., Yogi S., Nakatani N.: Antioxidative compounds isolated from Kokuto, noncentrifugal cane sugar. Bioscience, biotechnology, and biochemistry 1996;60:1714-1716.

[17] Takara K., Matsui D., Wada K., ICHIBA T., NAKASONE Y.: New antioxidative phenolic glycosides isolated from Kokuto noncentrifuged cane sugar. Bioscience, biotechnology, and biochemistry 2002;66:29-35.

[18] Payet B., Shum C. S. A., Smadja J.: Assessment of antioxidant activity of cane brown sugars by ABTS and DPPH radical scavenging assays: Determination of their polyphenolic and volatile constituents. J Agric Food Chem 2005;53:10074-10079. 
[19] Duarte-Almeida J. M. I. C., Salatino A., Genovese M. I. E. S., Lajolo F. M.: Phenolic composition and antioxidant activity of culms and sugarcane (Saccharum officinarum L.) products. Food Chem 2011;125:660-664.

[20] Duarte-Almeida J. M. I. C., Novoa A. V., Linares A. F., Lajolo F. M., Genovese M. I. E. S.: Antioxidant activity of phenolics compounds from sugar cane (Saccharum officinarum L.) juice. Plant Food Hum Nutr 2006;61:187-192.

[21] Colombo R., Lancas F. M., Yariwake J. H.: Determination of flavonoids in cultivated sugarcane leaves, bagasse, juice and in transgenic sugarcane by liquid chromatography-UV detection. J Chromatogr A 2006;1103:118-124.

[22] Nuissier G., Bourgeois P., Fahrasmane L., Grignon-Dubois M.: Evaluation of vinasses from sugarcane molasses distillation as a new source of sugarcane wax. Chem Nat Compd+ 2008;44:552555.

[23] Nuissier G., Bourgeois P., Grignon-Dubois M., Pardon P., Lescure M. H.: Composition of sugarcane waxes in rum factory wastes. Phytochemistry 2002;61:721-726.

[24] Feng S., Luo Z., Zhang Y., Zhong Z., Lu B.: Phytochemical contents and antioxidant capacities of different parts of two sugarcane (Saccharum officinarum L.) cultivars. Food Chem 2014;151:452-458.

[25] Slinkard K., Singleton V. L.: Total phenol analysis: Automation and comparison with manual methods. Am J Enol Viticult 1977;28:49-55.

[26] Yamaguchi T., Takamura H., Matoba T., Terao J.: HPLC method for evaluation of the free radical-scavenging activity of foods by using 1, 1-diphenyl-2-picrylhydrazyl. Bioscience, biotechnology, and biochemistry 1998;62:1201-1204.
[27] Miller N. J., Sampson J., Candeias L. P., Bramley P. M., RiceEvans C. A.: Antioxidant activities of carotenes and xanthophylls. Febs Lett 1996;384:240-242.

[28] Benzie I. F., Strain J. J.: The ferric reducing ability of plasma (FRAP) as a measure of "antioxidant power": The FRAP assay. Anal Biochem 1996;239:70-76.

[29] Shahidi F., Ho C.: Antioxidant measurement and applications: An overview, 2007, 956, pp 2-7.

[30] ALZOREKY N., NAKAHARA K.: Antioxidant activity of some edible yemeni plants evaluated by Ferrylmyoglobin/ABTS*+ assay. Food science and technology research 2001;7:141-144.

[31] Pulido R., Bravo L., Saura-Calixto F.: Antioxidant activity of dietary polyphenols as determined by a modified ferric reducing/antioxidant power assay. J Agric Food Chem 2000;48:3396-3402.

[32] Thaipong K., Boonprakob U., Crosby K., Cisneros-Zevallos L., Byrne D. H.: Comparison of ABTS, DPPH, FRAP, and ORAC assays for estimating antioxidant activity from guava fruit extracts. Journal of food composition and analysis 2006;19:669-675.

[33] Arnao M. B., Cano A., Acosta M.: The hydrophilic and lipophilic contribution to total antioxidant activity. Food Chem 2001;73:239244

[34] Awika J. M., Rooney L. W., Wu X., Prior R. L., CisnerosZevallos L.: Screening methods to measure antioxidant activity of sorghum (sorghum bicolor) and sorghum products. J Agric Food Chem 2003;51:6657-6662.

[35] Fu, X.,Yu, S.J. ,Min, Y.G., and Chung, C.C.: Extraction of natural antioxidants from sugarcane. Sugarcane and Canesugar2003; 5:3741. 